\title{
Choroidoretinal neovascularisation following radon seed treatment of retinoblastoma in two patients
}

\author{
D B Archer, W M K Amoaku, G Kelly
}

\begin{abstract}
Two patients who developed localised radiation retinopathy many years after brachytherapy for retinoblastoma are described. In both patients extracapsular cataract extraction and YAG laser capsulotomy were followed by preretinal and vitreous haemorrhage and in one patient there was deterioration of existing radiation retinopathy with macular oedema. Premacular and vitreous haemorrhage occurred from focal, preretinal neovascular membranes which appeared to originate from residual choroidal vascular radicals. Laser photocoagulation was successful in ablating preretinal neovascular membranes and limiting the extent of macular oedema from incompetent retinal capillaries adjacent to the atrophic macular scars.

(Brf Ophthalmol 1993; 77: 95-99)
\end{abstract}

Brachytherapy is an effective and firstline therapy for selected retinoblastomas, and where the macula and optic nerve are uninvolved the visual prognosis is typically good. ${ }^{1-4}$ Most brachytherapeutic schedules aim to deliver about $40 \mathrm{~Gy}$ of radiation to the tumour apex and 100 $160 \mathrm{~Gy}$ to the scleral base. ${ }^{3-5}$ The retinal vessels are judged to be at risk when the total dose of radiation exceeds $30 \mathrm{~Gy}$, although retinopathic changes are often slow to emerge and may not be obvious on routine clinical examination. All patients receiving brachytherapy for retinoblastoma develop retinal vascular changes at or adjacent to the tumour site, although the degree and extent of retinopathy are highly variable and visual functions may only be affected significantly if the macula is implicated in the disease process. ${ }^{2-4}$

A proportion of patients receiving brachytherapy for retinoblastomas, particularly those receiving multiple treatments for equatorial or peripheral tumours, develop lens opacities, although the cataract is typically slow to develop and may not present for years after the initial tumour therapy. ${ }^{1-36}$ Where retinoblastomas are treated by combined brachytherapy and teletherapy, there is a higher incidence and early appearance of cataract and increased likelihood of significant radiation retinopathy.'

The literature records instances of successful cataract extraction and visual recovery in patients who received teletherapy for intraocular and extraocular tumours, ${ }^{7-10}$ and brachytherapy for retinoblastomas and choroidal melanomas, ${ }^{112}$ and in most instances any existing retinopathy did not deteriorate measureably..$^{92}$ There are, however, no references to the effects of cataract extraction, intraocular lens implantation, and YAG laser capsulotomy on any underlying retinopathy associated with brachytherapy for retinoblastomas.

We document two patients who received radium seed therapy for isolated retinoblastomas and subsequently developed lens opacities and unique chorioretinal vascular changes. Cataract extraction, intraocular lens implantation, and YAG laser capsulotomy had an adverse effect on the underlying, radiation induced retinal vasculopathy.

\section{Materials and methods}

\section{CASE 1}

This female patient was diagnosed as suffering from bilateral retinoblastomas in 1952, at the age of 2 years and 4 months. The right eye was enucleated (22 February 1952), and two radon seeds secured to the left inferior sclera (13 March 1952) at the site of localised retinoblastomas and left in place for 4 days. Sections of the enucleated right eye confirmed the presence of a poorly differentiated 'retinoblastoma exophytum', but without invasion of the optic nerve. Two additional radon seeds were attached to the left inferior sclera on 24 May 1952 for 5 days, and on 28 August 1952 for a further 5 days. Subsequently, there was striking atrophy of the tumour mass. In 1954 and 1956, however, retinal haemorrhages were recorded adjacent to the radiation scars. The haemorrhages resolved slowly without event. In 1956 segmental posterior cortical cataract was noted, but acuity still improved to $6 / 12(+3.50 \mathrm{DS})$ and remained at this level until 1965 . Thereafter visual acuity slowly declined and in 1986 was recorded as $6 / 24$ $(+0.50 \mathrm{DS} /-2.00 \mathrm{DC} \times 150)$. At that time the patient was documented as having $4+$ posterior subcapsular lens opacities. A large inferotemporal chorioretinal scar was noted in addition to an area of peripapillary chorioretinal atrophy. No radiation retinopathy was observed.

Left extracapsular cataract extraction with posterior chamber lens implantation was carried out on 14 July 1986 which improved acuity to $6 / 9(+1 \cdot 25 \mathrm{DS} /-2 \cdot 75 \mathrm{DC} \times 20)$. Postoperative evaluation revealed an intact posterior capsule with some eccentric opacification. Ten days post-surgery visual acuity declined to $6 / 24$. Fundus examination showed no additional abnormalities; however, significant posterior capsular haze was noted. The patient was prescribed drops containing dexamethasone, neomycin, hypromellose, and polymyxin B (Maxitrol) four times daily and YAG laser capsulotomy undertaken on 29 July 1986 . This produced a satisfactory clear, central aperture. Acuity improved to 6/18 and Maxitrol medication was continued for 6 weeks. Vision remained 
steady until May 1989 when it deteriorated slowly, but significantly, over a 5 month period.

On examination on 26 October 1989 acuity registered 2/60 left and the axial mediae were clear. An inferior full thickness chorioretinal scar extended from the ora serrata to within two disc widths of the optic nerve head and had a sizeable 'neovascular complex' within its posterior substance (Fig 1A). There were striking drusen of the optic disc. Microvascular abnormalities extended from the temporal margin of the scar to involve the inferior macula and there was pronounced exudation and fluid accumulation at and beyond the macular region (Fig 1B). The exudates were located deep within the retina. Subtle capillary defects were also present superior and nasal to the chorioretinal scar and associated with intraretinal exudation.

Angiography confirmed the presence of choroidoretinal communications and neovascular membranes arising from residual choroidal vessels at the inferior chorioretinal scar. At the temporal margin of the scar there were dilated capillaries, intraretinal microvascular abnormalities, and evidence of inner retinal ischaemia at the inferior macula. Some of the major retinal vessels adjacent to the treatment scar were severely constricted, sheathed, or occluded (Fig 2). The abnormal vessels leaked and stained with dye in the late angiographic frames. Capillary abnormalities and drusen at the optic disc fluoresced brightly in late angiography.

Argon laser photocoagulation was carried out to the region of incompetent vessels which juxtaposed the radiation scar and directly to the area of the neovascular membranes on three occasions. Haemorrhage, fluid, and exudates gradually absorbed with improvement of visual acuity. At final review (5 March 1992) there was almost complete absorption of haemorrhage and exudates, and acuity improved to 6/18 part (Fig 3). Reading vision was N/5 with $+6.00 \mathrm{DS}$ at 9 inches. A small, isolated, residual preretinal haemorrhage remained within the body of the scar, but this was not associated with any neo- vascular membrane or abnormal vascular complexes. Fluorescein angiography confirmed that the macular and perifoveolar microvasculatures were intact and competent and that the focal retinopathy temporal to the radiation scar had atrophied in part following laser photocoagulation (Fig 4). All choroidoretinovitreal neovascular membranes had resolved, although residual choriocapillaris units within the chorioretinal scar fluoresced brightly in the later phase angiograms. The posterior vitreous face was detached.

\section{CASE 2}

This male patient was diagnosed as suffering from bilateral retinoblastomas in 1952. His left eye was enucleated at age 18 months, and discrete tumours, superior to the right macula, were treated with radon seeds. The tumours atrophied and were replaced by full thickness chorioretinal scars, superior to the main superotemporal vascular arcades and extending to the ora serrata. Acuity remained $6 / 5$ unaided in the right eye.

In 1970 the patient developed sectorial posterior subcapsular lens opacities which gave symptoms of glare but did not substantially affect acuity. Symptoms responded to drops of pilocarpine $0.5 \%$ three times daily. Examination in 1977 revealed a focus of new vessels at the centre of the atrophic scar but identified no evidence of tumour recurrence. The lens opacities slowly progressed and by 11 June 1990 acuity was 6/36 (N18). Examination of the fundus at this time only revealed an atrophic chorioretinal scar with no evidence of radiation retinopathy or residual new vessels.

Right extracapsular cataract extraction with intraocular lens implantation was carried out on 13 June 1990 . There was dense opacification and fibrosis of the posterior lens capsule which was resistant in part to intraoperative debridement. Two weeks postoperatively a large preretinal haemorrhage was observed at the radiation scar, together with an area of exudation. Opacification
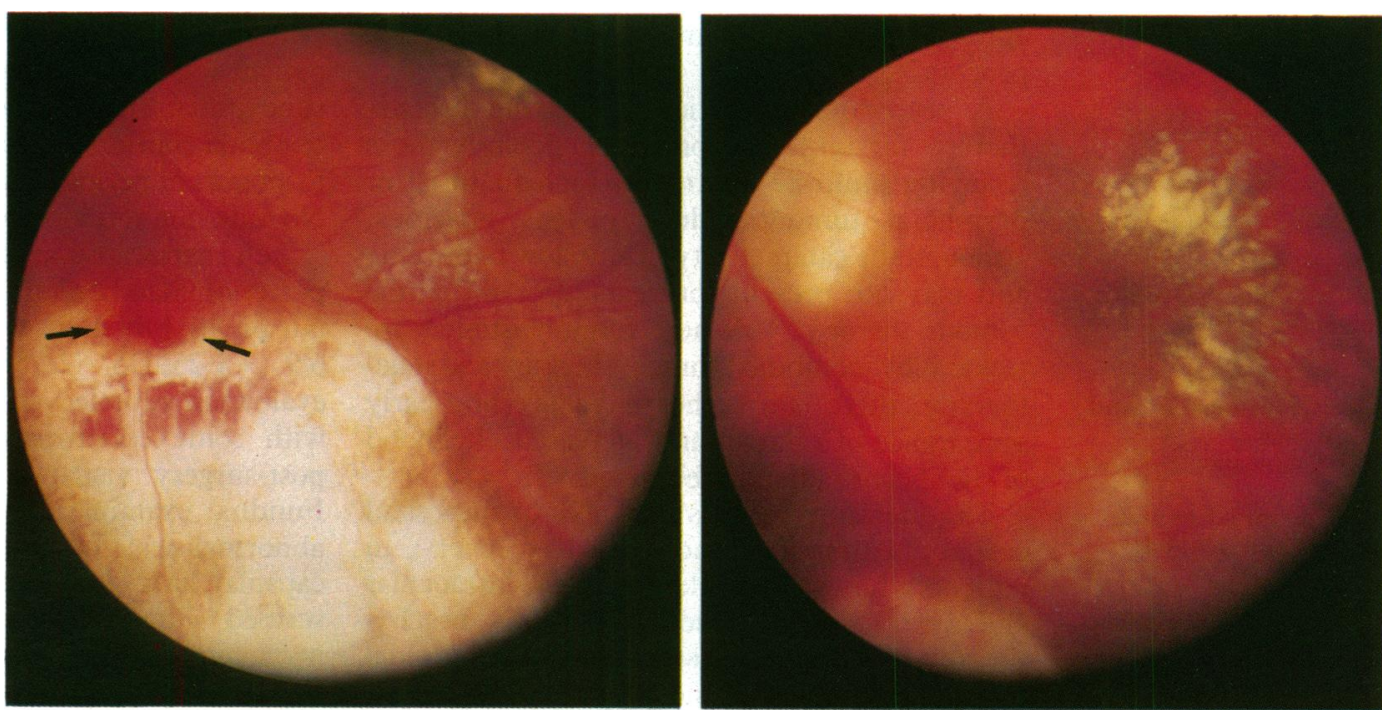

Figure 1 (Case 1-October 1989). (A) Dense segmental chorioretinal radiation scar inferior to the left optic disc. Intraretinal and preretinal haemorrhage have occurred at and beyond two discrete neovascular membranes (arrows). A sheathed inferotemporal vein traverses ischaemic retina. $(B)$ There is extensive fluid accumulation and exudation at the left macula (visual acuity $2 / 60$ ). 


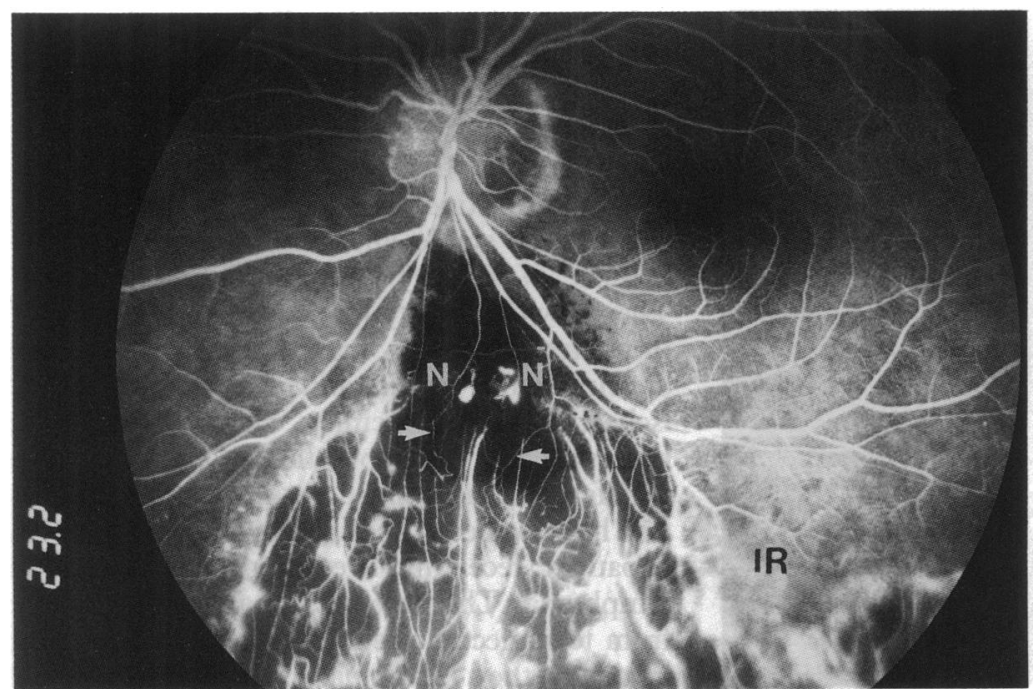

Figure 2 (Case 1-October 1989). Venous phase angiogram. Two hyperfluorescent choroidoretinal neovascular membranes $(N)$ originate from the posterior aspect of the radiation scar. Microaneurysms, dilated capillaries, ischaemic inner retina $(I R)$, and chorioretinal communications (arrows) are present at and beyond the scar. Incompetent residual choriocapillaris units are hyperfluorescent.
Figure 3 (Case 1-March 1992). Macular oedema, exudates, and scar haemorrhages have resolved (visual acuity 6/18-). Optic disc drusen are more prominent and discrete. of the posterior capsule intensified over a period of 4 months and YAG laser capsulotomy was undertaken on 9 October 1990 . Acuity improved to $6 / 6(\mathrm{~N} / 5)$ in the right eye, although preretinal haemorrhage persisted at the radiation scar. Retinal exudation had partially resolved and no retinal tears were identified. Fluorescein angiography (May 1991) outlined a complex of new vessels at the chorioretinal scar, which profusely leaked dye in the late angiographic frames. In June 1990 the patient had a 'superficial spreading melanoma' excised from the region of the right nipple.

At final review (5 March 1992) visual acuity was recorded as $6 / 6(\mathrm{~N} / 5)$. The area of exudation at the plaque scar (4 disc diameters superior to the right foveola) had largely resolved, although a sizeable area of preretinal haemorrhage persisted at the scar. Dilated, tortuous vessels at the site of haemorrhage were associated with discrete choroidovitreal neovascular membranes (Fig 5). Fluorescein angiography confirmed the choroidal origin of the neovascular complexes. There was anterior displacement of the posterior vitreous face.

\section{Discussion}

Brachytherapy is an effective treatment for retinoblastoma, particularly where the tumour is small, discrete, and remote from the macula and optic nerve head. ${ }^{134}$. The retina and choroid overlying and adjacent to the site of the radioactive plaque or seed application become grossly atrophic with attenuation or disappearance of most retinal and choroidal vascular radicals and subtotal loss of retinal substance. ${ }^{36}$ The retinal vasculature adjoining the radiation scar may display a spectrum of changes, including microaneurysms, dilated and incompetent vessels, and varying degrees of capillary atrophy and closure. ${ }^{31314}$

In general, radiation retinopathy subsequent to brachytherapy extends only a short distance beyond the margin of the atrophic radiation scar, and where the lesions are extramacular central visual functions typically remain good. ${ }^{31314}$ In contrast to retinopathy associated with teletherapy, the retinal vascular lesions induced by radioactive plaque therapy show little evidence of progression and the discrete areas of ischaemic retina are rarely associated with preretinal neovascularisation. ${ }^{31314}$

The two patients described in this report were typical in that they displayed stable, discrete, atrophic chorioretinal scars at the site of radon seed therapy and demonstrated no significant or identifiable vasculopathy in the adjoining retina. In each instance the chorioretinal scars were extramacular and good central visual functions were maintained until lens opacities developed. Cataracts were identified within a few years of therapy; however, progression was slow, ${ }^{6}$ and it was not until 35 years or so post-therapy that deterioration of vision dictated cataract extraction. Patients developing cataract following brachytherapy for choroidal melanomas do so more rapidly, probably reflecting higher doses of radiation to the eye..$^{15}$

Cataract surgery in both patients was associated with the development of preretinal or vitreous haemorrhage at the radiation scar from choroidoretinal vascular abnormalities. In patient 2 there was also a profuse outpouring of opalescent fluid in the region of the scar, which was probably a fibrin-rich exudate from incompetent vessels at or adjacent to the irradiated retina and choroid. This elevated opaque lesion initially raised the possibility of local recurrence of tumour, ${ }^{17}$ but it gradually disappeared with time and was not linked to the original neoplasm.

Some of the vascular abnormalities detected within the atrophic radiation scar had the features of choroidoretinal communications or choroidoretinovitreal neovascular membranes. Both vascular anomalies filled rapidly with dye from the choroidal circulation; however, the choroidoretinal communications remained competent, whereas the neovascular membranes profusely leaked dye. The choroidovitreal neovascular complexes were discrete and, although of varying calibre, had an angiomatous-like quality to their vitreoretinal component. These neovascular membranes expanded slowly and ramified at the level of the internal limiting membrane, appearing to gain attachment to the 


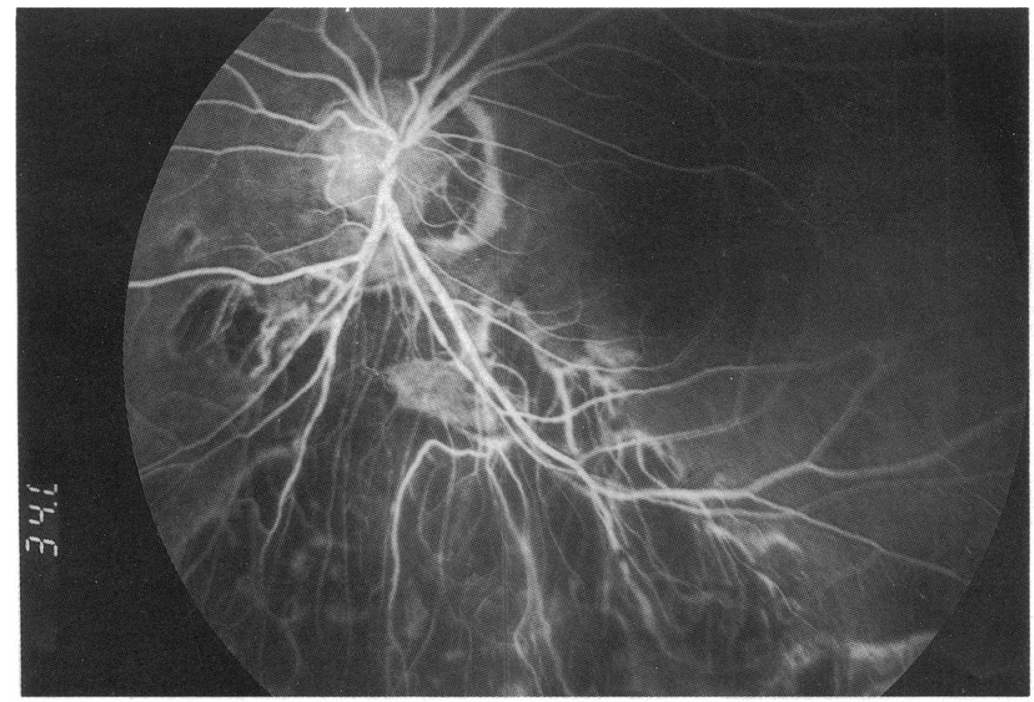

Figure 4 (Case 1-March 1992). Venous phase angiogram. The neovascular membranes have atrophied and there is no significant dye leakage from inferior macular capillaries. The disc drusen are hyperfluorescent.

Figure 5 (Case 2-March 1992). Sectorial radiationchorioretinal scar, superotemporal fundus right eye. Two neovascular membranes originating from the residual choroidal circulation are present (arrows). Preretinal and vitreous haemorrhage and plasma-like exudate $(P)$ are resolving. posterior vitreous face. Detachment of the posterior vitreous face following cataract surgery and YAG laser capsulotomy ${ }^{18} 19$ is the most likely cause of their disruption and subsequent preretinal and vitreous haemorrhage. Haemorrhage into the vitreous body in patient 2 cleared relatively rapidly; however, preretinal haemorrhage at the chorioretinal scars in both patients remained unchanged for many months, reflecting the attenuated circulation and limited phagocytic activity in this region.

Egbert $e t a l^{20}$ described vitreous haemorrhage in some of their patients receiving teletherapy for retinoblastomas. They believed the haemorrhage arose from unsupported tumour vessels in 'shrinking tumour'; however, these haemorrhages were noted soon after radiation retinopathy and probably have different origins from those described in this report.

Choroidoretinal vascular communications occur commonly within chorioretinal scars and are most often observed in longstanding disciform scars associated with age-related macular degeneration. ${ }^{21}$ They are a component of the organisational process, but remain competent and do not adversely affect the neighbouring retina or require treatment. Choroidoretinovitreal neovascular complexes also occur at sites of chorioretinal atrophy and may follow intense photocoagulation of attenuated ischaemic retina

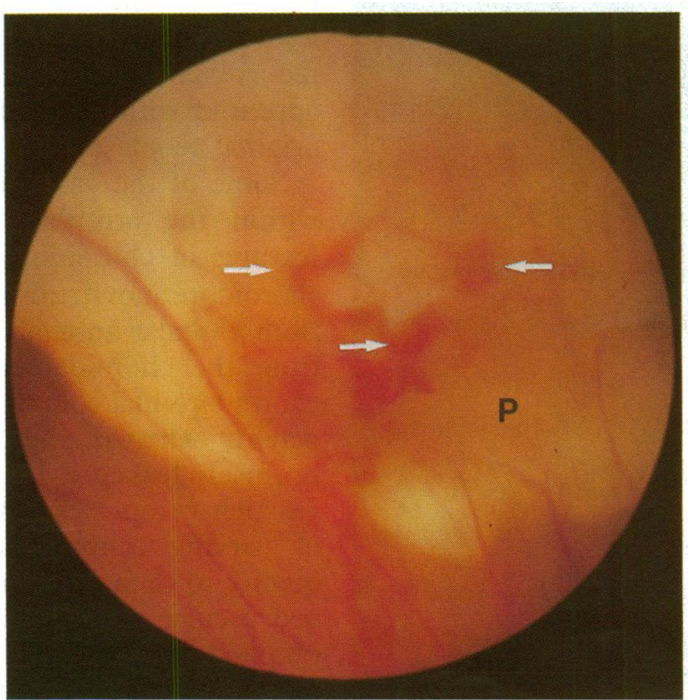

- for example, in sickle cell retinopathy, ${ }^{22}$ and branch retinal vein occlusion. ${ }^{23}$ Such membranes are often profuse, haemorrhagic and resistant to treatment. Choroidoretinovitreal vascular abnormalities that develop following brachytherapy for retinoblastomas are small, discrete, and appear stable and unsymptomatic in the absence of surgery and vitreous detachment. Their low propensity for growth and invasion of the vitreous cavity may reflect the absence of circulatory stasis and widespread retinal ischaemia in their vicinity, which is considered necessary for the elaboration of putative vasoproliferative factors. Subretinal neovascularisation has also been described by Boozalis $e t \mathrm{al}^{24}$ in a patient receiving teletherapy for an orbital lymphoma. The new vessels appeared to arise from telangiectatic retinal vascular radicals and invaded the subretinal space. Histological sections, however, showed that these vessels had no communication with the choroidal circulation and appeared different from those described in this communication.

Patient 1 also developed severe macular oedema and exudation shortly after cataract extraction and capsulotomy, with consequential decline in visual acuity. The precise factors initiating extravasation of fluid and exudation into the macula in this instance are not clear. There was, however, angiographic evidence of radiation retinopathy adjacent to the chorioretinal scar and inferior macula, and it may be that altered haemodynamic events at or after cataract surgery caused or exaggerated incompetence of these abnormal capillaries with accumulation of fluid at the macula. It may also be that fluid and blood constituents from the neovascular membranes and residual choriocapillaris units at the atrophic scar permeated the macula via the temporal margin of the scar where cellular barriers were non-existent or defective. The foveal microvasculature and retinal pigment epithelium appeared clinically normal; nevertheless, the gratuitous extracellular fluid at the macula clearly overwhelmed the normal retinal pump mechanisms in this region and resulted in chronic macular oedema with reduction of visual functions. ${ }^{25}$ Whether inflammation, induced by surgery, contributed to the development of maculopathy is uncertain, but the absence of observable vitritis, perifoveolar vasculitis, or frank cystoid macular changes would suggest that inflammation was not an important component of the maculopathy. The absence of maculopathy in patient 2 was probably a reflection of the distance of the radiation scar from the macula.

Irrespective of the source of fluid, meticulous photocoagulation to areas of residual retinopathy and choroidovitreal neovascular membranes was successful in aiding resolution of macular oedema and vitreous haemorrhage. Photocoagulation may become necessary in patient 2 for the choroidal vitreal membrane, although at present the macula is not considered under threat, and an expectant management policy is being pursued. Kinyoun $e^{2} \mathrm{al}^{26}$ demonstrated the effectiveness of laser photocoagulation in containing both macular oedema and vitreous haemorrhage in patients with maculopathy and 
proliferative disease secondary to radiation. This report describes a further type of radiation retinopathy which is amenable to laser photocoagulation.

In general most patients receiving brachytherapy for small or moderate size retinoblastomas only develop discrete areas of radiation retinopathy limited to the margins of the radiation scar. ${ }^{314}$ Such retinopathy is not associated with neovascular membranes, and produces few or no observable abnormalities following routine cataract extraction. In some patients, however, as illustrated in this report, cataract extraction and capsulotomy may destabilise existing residual radiation retinopathy, causing vitreous haemorrhage and macular oedema where lesions are perimacular. Microvascular abnormalities may be obscured by lens opacities and remain untreated at preoperative assessment. Such patients should be counselled about the possibility of vitreous haemorrhage, or macular oedema postextraction or capsulotomy. In the event of haemorrhage or macular oedema laser photocoagulation may have beneficial effects on the disease process.

1 Amendola BE, Lamm FR, Markoe AM, Karlsson UL, Shield J, Shields CL, et al. Radiotherapy of retinoblastoma. A J, Shields CL, et al. Radiotherapy of retinoblastoma. A review of 63 children treated with

2 Migdal C. Bilateral retinoblastoma: the prognosis for vision. Br F Ophthalmol 1983; 67: 592-5.

3 Ehlers N, Kaae S. Effects of ionising radiation on retinoblastoma and on the normal ocular fundus in infants. A photographic and fluorescein angiographic study. Acto Ophthalmol 1987; 181 (suppl): 3-84.

4 Shields JA, Giblin ME, Shields CL, Markoe AM, Karlsson U, Brady LW, et al. Episcleral plaque radiotherapy for retinoblastoma. Ophthalmology 1989; 96: 530-7.

5 Shields JA. Retinoblastoma. In: Shields JA, ed. Diagnosis and management of intraocular tumours. St Louis: Mosby, 1983: managemen.

6 Merriam GR, Jr, Szechter A, Focht EF. The effects of ionising radiation on the eye. Front Radiat Ther Oncol 1972; 6: 34685.
7 Monge OR, Flage T, Hatlevoll R, Vermund H. Sight saving herapy in retinoblastoma: experience with external megavoltage radiotherapy. Acta Ophthalmol 1986; 64: 414-20.

8 Haye C, Desjardins L, Elmaleh C, Schlienger P, Zucker JM Laurent $M$. Prognosis and treatment of retinoblastoma. Ophthalmic Paediatr Genet 1989; 10: 151-5.

9 Amoaku WMK, Archer DB. Cephalic radiation and retinal vasculopathy. Eye 1990; 4: 195-203.

10 Brooks HL, Meyer D, Shields JA, Balas AG, Nelson LB Fontanesi J. Removal of radiation-induced cataracts in patients treated for retinoblastoma. Arch Ophthalmol 1990; patients treated $1701-8$.

11 Augsburger JJ, Shields JA. Cataract surgery following cobalt60 plaque radiotherapy for posterior uveal malignan melanoma. Ophthalmology 1985; 92: 815-22

12 Fish GE, Jost BF, Snyder WB, Fuller DG, Birch DG. Cataract extraction after brachytherapy for malignant melanoma of the choroid. Ophthalmology 1991; 98: 619-22.

13 Stallard HB. Radiant energy as (a) a pathogenic (b) a therapeutic agent in ophthalmic disorders. Brf Ophthalmo Monograph Suppl) 1933; 6: 1-126.

14 Brown GC, Shields JA, Sanborn G, Augsburger JJ, Savino PI, Schatz NJ. Radiation retinopathy. Ophthalmology 1982; 89: 1494-501.

15 Beitler JJ, McCormick B, Ellsworth RM, Abramson DH Anderson LL, Loffredo C. Ocular melanoma: total dose and dose rate effects with Co60 plaque therapy. Radiology 1990 176: $275-8$.

16 Packer S. Iodine-125 radiation of posterior uveal melanoma. Ophthalmology 1987; 94: 1621-6.

17 Elmassri A. Radiation chorioretinopathy. Br $\mathcal{F}$ Ophthalmol 1986; 70: 326-9.

18 Macewen CJ, Baines PS. Retinal detachment following YAG laser capsulotomy. Eye 1989; 3: 759-63.

19 McDonnell PJ, Patel A, Green WR. Comparison of intracapsular and extracapsular cataract surgery. Histopathologic study of eyes obtained post mortem. Ophthalmology 1985 92: 1208-25.

20 Egbert PR, Donaldson SS, Moazed K, Rosenthal AR. Visual results and ocular complications following radiotherapy for retinoblastoma. Arch Ophthalmol 1978;96: 1826-30.

21 Green WR, Key SN III. Senile macular degeneration: histological study. Trans Am Ophthalmol Soc 1977; 75: 180 254

22 Fox PD, Acheson RW, Sergeant GR. Outcome of iatrogenic choroidal neovascularisation in sickle cell disease. Brf Ophthalmol 1990; 74: 417-20.

23 Archer DB, Gardiner TA. Morphologic, fluorescein angiographic and light microscopic features of experimental choroidal neovascularization. Am $\mathcal{F}$ Ophthalmol 1981; 91: 297-311.

24 Boozalis GT, Schachat AP, Green WR. Subretinal neovascuarization from the retina in radiation retinopathy. Retina 1987; 7: 156-61.

25 Bradford JD, Wilkinson CP, Bradford RH, Jr. Cystoid macular oedema following extracapsular cataract extraction and posterior chamber intraocular lens implantation. Retina 1988; 8: $161-4$.

26 Kinyoun JL, Chittum ME, Wells CG. Photocoagulation treatment of radiation retinopathy. Am $\mathcal{F}$ Ophthalmol 1988; 105: 470-8. 